\title{
Depression, Anxiety, and Stress during COVID-19 Epidemic among Public Health Students in Thailand
}

\author{
Nirachon Chutipattana, Cua Ngoc Le* and Supreecha Kaewsawat
}

\begin{abstract}
Excellent Center of Dengue and Community Public Health, School of Public Health, Walailak University,
\end{abstract} Nakhon Si Thammarat 80160, Thailand

('Corresponding author's e-mail: cua.le@wu.ac.th)

Received: 17 April 2021, Revised: 18 July 2021, Accepted: 28 July 2021

\begin{abstract}
By the end of March 2020, the COVID-19 epidemic were widespread in Thailand led to the closures of schools and universities. The home quarantine, social distancing, and other restrictions are likely to have psychological impact on students. This study aimed to estimate the prevalence of depression, anxiety, and stress and determine predictors among undergraduate public health students. A crosssectional study was conducted at the School of Public Health - Walailak University. Research subjects included 463 students selected by a 2-stage random sampling. A research tool was a self-administered standardized 21-item depression, anxiety, and stress scale. An ordinal logistic regression analysis was executed to determine the predictors of depression, anxiety, and stress. The results showed that the prevalence of depression, anxiety, and stress was $24.8,31$, and $18.8 \%$, respectively. Significant predictors of depression and anxiety were educational environment $(\mathrm{OR}=0.35,95 \% \mathrm{CI}: 0.13-0.6 ; \quad \mathrm{OR}=0.3,95 \%$ CI: 0.2 - 0.7), and tense feelings during learning (OR = 3.57, 95\% CI: $1.9-6.6$; OR = 0.5, 95\% CI: 0.2 $0.8)$. Relationship with boy/girlfriends was considered a predictor of depression and stress $(\mathrm{OR}=2.5$, 95\% CI: 1.2 - 4.9; OR = 0.5,95\% CI: $0.2-0.9)$. Marital status of parent (OR = 15.5, 95\% CI: $1.2-192.3)$ and family provision for learning $(\mathrm{OR}=3.1,95 \% \mathrm{CI}: 1.3-7.6)$ were predictors of depression, and specialized majors $(\mathrm{OR}=0.2,95 \% \mathrm{CI}: 0.1-0.7)$ was a predictor of stress. Noticeable worries about "My physical health", "Health systems being overloaded", and "Restricted liberty of movement" are also considered as predictors of depression, anxiety, and stress. The findings are needed to develop a good education environment and counseling services for cases having emotional problems.
\end{abstract}

Keywords: Depression, Anxiety, Stress, COVID-19, Public health student

\section{Introduction}

Depression and other common mental disorders have been wide-spreading and become the challenges of global mental health. The World Health Organization (WHO) estimated that depression and anxiety accounted for $4.4 \%$ and $3.6 \%$ of the global population in 2015, respectively [1]. According to WHO, depression is a common mental disorder characterized by persistent sadness, a lack of interest or pleasure in previously enjoyable activities, disturbed sleep, appetite, tiredness, and poor concentration [2]. Anxiety is a state of uneasiness. It is a bodily response to a perceived danger that could be real or imaginary and triggered by an individual's thoughts, beliefs, and feelings [3]. Finally, stress is a body's reaction to a challenge or demand, a feeling of emotional or physical tension that results from any event or thought, and affects an individual's physical and psychological well-being [4]. Moreover, depression, anxiety, and stress (DAS) are also the most common psychiatric problems among university undergraduate students [5,6]. Students with DAS are likely to suffer from academic difficulties, dropouts, relationship disturbance with friends and family members, and failure to cope with the anxious situation [7,8]. A study in Australia showed that $13.53 \%$ of students had psychological morbidities [9]. In Turkey, depression, anxiety, and stress accounted for 27.1, 47.1, and 27\% among students, respectively [5]. For health profession's students (HP students), common mental health problems affected a significant proportion of students [10-12]. Depression in HP students is higher than the age-matched population $[6,13,14]$, such as the $52.4 \%$ pharmacy students at a United States school [15]. A recent meta-analysis included 77 studies show that the global depression rate of medical students is $28.0 \%$, $(95 \%$ confidence interval: $24.2-32.1 \%$ ) [16]. Students with DAS lead to a lack of self-confidence, and the ability to cope 
with daily life problems that directly affect the academic performance of students $[17,18]$. As mentioned, their coping abilities with anxious situations, including environmental factors like emerging epidemics, may contribute to DAS in these students [19].

In December 2019, the novel coronavirus epidemic (COVID-19) broke out in Wuhan, which has spread rapidly to all provinces in China and many countries [20,21]. In Thailand, the $1^{\text {st }}$ COVID-19 case was a Wuhan resident to travel to Bangkok detected on $8^{\text {th }}$ January, 2020. By the end of March, 60 of Thailand's 77 provinces had reported cases and the epidemic were widespread [22]. To reduce the spread of COVID-19, all schools, colleges, and universities have closed since. This situation may hinder university students' studies, disrupt their daily routines and habits, and impact their mental health. Further, home quarantine, physical/spatial distancing, and other restrictions are likely to have psychological impact on students [23] and negatively influence their mental well-being [24]. Almost all college and university students in Thailand were asked to stay home and isolate themselves lasted for a longer time, which might increase the risk of depressed mood [25]. A study in Saudi Arabia has shown that dental students from different universities acquiring high levels of DAS accounted for 60.64, 37.02, and 34.92\%, respectively during the spread of COVID-19 [26]. In Wuhan and Beijing, China, the prevalence of anxiety disorder was $17.1 \%$ and depression was $25.3 \%$ among medical students during COVID-19 pandemic outbreak [27]. Uses of PubMed, ERIC and Google Scholar can easily find many studies that estimate the prevalence of DAS involved students of different heath disciplines such as medicine, dentistry, pharmacy and nursing before and during COVID-19 epidemic outbreaks [6,8,13,15$18,26,27]$. However, there are no studies on DAS related to public health students even though they are the future source of labor for health development in each country. Therefore, it is necessary to assess current levels of DAS and determine predictors among public health students, especially during COVID19 epidemic to prevent DAS from affecting their academic performance and career development. To authors' knowledge, no studies have investigated the prevalence of DAS among public health students during COVID-19 epidemic in Thailand. Hence, this study investigated psychological problems that undergraduate public health students of Walailak University have experienced during the COVID-19 outbreaks in Nakhon Si Thammarat, Thailand.

\section{Materials and method}

\section{Study design, participants, and sampling procedures}

A cross-sectional study was conducted among undergraduate public health students at Walailak University in Nakhon Si Thammarat province, southern Thailand after an outbreak of COVID-19. The duration of the study was 4 months from August to December 2020. All undergraduate public health students from the $1^{\text {st }}$ year to $4^{\text {th }}$ - year class were eligible to participate for a total of 881 students. Students were excluded from the study if anyone was receiving any cognitive or behavioral therapy, medication, or the combination. The study was conducted in accordance with the Institutional Research Ethics and the Helsinki declaration. All procedures were approved by the Walailak University Ethics Research Commission (Ref: WUEC-20-156-01).Survey data were collected anonymously and participants agreed on responses through informed consent. The sample size was calculated based on the prevalence of $50 \%$ and using the standard formula of prevalence as follows:

$\mathrm{N}=\mathrm{z}^{2} \mathrm{p}(1-\mathrm{p}) / \mathrm{d}^{2}$

$\mathrm{Z}$ is the value from the standard normal distribution to reflect the confidence level. $\mathrm{Z}=1.96$ at $95 \%$ of the confidence interval with acceptance sample error 5\%. Prevalence of DASamong public health students was not available in previous studies so the value of $\mathrm{p}=0.5$ can be used to generate the largest sample size [28]. Sample size plus sample loss was rounded to 500. A total of 463 students were included in the study after data cleaning. The sample was selected by a 2-stage sampling technique. Public health classes were stratified into 4 strata $\left(1^{\text {st }}, 2^{\text {nd }}, 3^{\text {rd }}\right.$, and $4^{\text {th }}$-year classes $)$. Students from each stratum(class)were selected randomly. The probability proportional to size sampling was used to determine the number of students at each class with the random selection from the list of students.

\section{Data collection}

A 5-part, self-administered questionnaire composed of 56 close-ended questions. Part A included 10 questions related to sociodemographic characteristics such as age, gender, marital status, accommodation, year of study, academic grading, and friend relationship. Part B (6 questions) focused on family information associated with parent's marital status, family provision for learning, feeling in living with 
family, parent expectation and attention in the study. Part C (5 questions) and D (14 questions) were designed to gather information related to the learning process and worries in the context of the COVID-19 epidemic, respectively. In part D, students answered questions scored on a Likert scale (from 1 to 5) assessing levels of worries [29]. Finally, part E was the 21-item Depression, Anxiety and Stress Scales developed by Lovibond's brothers, including 3 sub-scales: Depression (DASS21-D), Anxiety (DASS21A), and Stress (DASS21-S) [30]. In this study, the Thai version DASS-21 was translated from the English version by the National Centre in HIV Epidemiology and Clinical Research in Australia [31]. Each subscale had 7 items. Every item comprised a statement and 4 options on 4 point-Likert scale to reflect severity levels as follows: 0 (Did not apply to me at all), 1 (Applied to me to some degree, or some of the time), 2(Applied to me to a considerable degree or a good part of the time) and to 3 (Applied to me very much, or most of the time). To yield equivalent scores to full DASS-42, the total score of each scale was multiplied by 2 and ranged from 0 to 42 points. Lovibond and Lovibond [30] recommended thresholds for normal, mild, moderate, severe and extremely severe levels of DAS as follows: Normal (depression: 0-9, anxiety: 0-7, and stress: 0 - 14); mild (depression: 10-13, anxiety: 8-9, and stress: 15-18); moderate (depression: 14-20, anxiety: 10-14, and stress:19-25); severe (depression: 21-27, anxiety: 15-19, and stress: 26-33); extremely severe (depression: $\geq 28$, anxiety: $\geq 20$, and stress: $\geq 34$ ). The content validity of questionnaires of worry in the COVID-19 context and DASS-21 was assessed by 3 experts through the Content Validity Index to be 0.93 and 0.95 , respectively. Regarding the reliability test, test-retest reliability of worry in COVID-19 context and DASS scales showed the Pearson correlation coefficient $\mathrm{r}=$ $0.813(\mathrm{p}=0.001)$ and $\mathrm{r}=0.921(\mathrm{p}=0.001)$, respectively (measuring among 15 students in 2 times, 2 weeks apart). Measurement scores in 2 times were highly correlated to demonstrate the stability of scales over time. The measure of internal consistency reliability was conducted on a sample of 30 students to measure Cronbach's alpha. The overall Cronbach's alpha coefficients for worry and DASS-21 were 0.89 and 0.96, respectively. The Cronbach's alpha for the DASS21-D, DASS21-A, and DASS21-S subscales were 0.83, 0.78 , and 0.87 , respectively. Based on a cut-off point of 0.7 [32], it indicated high internal consistency reliability for worry scale in COVID-19 context, overall DASS-21, and subscales.

\section{Data analysis}

Data were cleaned and outliers were removed before analysis using SPSS version 20. Descriptive analysis showed the frequency, percentage of qualitative variables (all variables in parts A, B, and C except age), and the mean, and standard deviation of quantitative variables (age, all variables in parts D, and $\mathrm{E}$ ) in the self-administered questionnaires. Before performing inferential statistics techniques, the Kolmogorov-Smirnov test proved that all continuous variables were not normally distributed with $\mathrm{p}=0.0001$. Therefore, non-parametric methods such as Kruskal-Wallis and Mann-Whitney U tests were used to test the differences in the median of depression, anxiety, and stress symptom scores by sociodemographic and personal characteristics. Ordinal logistic regression analysis was performed to determine predictors of DAS from variables with statistically significant relationships with DAS as demonstrated by non-parametric tests. Statistical significance at an alpha $(\alpha)$ level equivalent to 0.05 was considered for all tests.

\section{Results and discussion}

\section{Results}

Sociodemographic, economic, and personal characteristics of students and their families

A total of 463 students completed the self-administered questionnaires with the response rate of $92.6 \%$. Their mean age was $19.85 \pm 1.38$ years, and the majority of students were female $(88.3 \%)$ and chose the major of community public health (56\%). Most of the students lived in university dormitories $(89.2 \%)$. The percentage of the good relationship with close friends and boy/girlfriends were $86.8 \%$, and $45.6 \%$, respectively. The results are summarized in Table 1. 
Table 1 Descriptive statistics for sociodemographic and personal characteristics.

\begin{tabular}{|c|c|c|}
\hline Variables & $\begin{array}{c}\text { Frequency } \\
\text { (n) }\end{array}$ & $\begin{array}{c}\text { Percentage } \\
(\%)\end{array}$ \\
\hline \multicolumn{3}{|l|}{ Gender } \\
\hline Male & 54 & 11.7 \\
\hline Female & 407 & 88.3 \\
\hline \multirow{2}{*}{ Age } & Mean \pm SD & \\
\hline & $19.85 \pm 1.38$ & \\
\hline \multicolumn{3}{|l|}{ Ethnics } \\
\hline Thai & 463 & 100.0 \\
\hline \multicolumn{3}{|l|}{ Marital status } \\
\hline Single & 463 & 100.0 \\
\hline \multicolumn{3}{|l|}{ Year of study } \\
\hline $1^{\text {st }}$-year & 153 & 33.2 \\
\hline $2^{\text {nd }}-$ year & 98 & 21.3 \\
\hline $3^{\text {rd }}$-year & 141 & 30.6 \\
\hline $4^{\text {th }}$-year & 69 & 15.0 \\
\hline \multicolumn{3}{|l|}{ Specialized majors } \\
\hline Community public health & 178 & 56.0 \\
\hline Occupational health & 76 & 23.9 \\
\hline Environmental health & 64 & 20.1 \\
\hline \multicolumn{3}{|l|}{ Relationship with close friends } \\
\hline Good & 387 & 86.8 \\
\hline $\mathrm{Bad}$ & 2 & 0.4 \\
\hline Normal & 57 & 12.8 \\
\hline \multicolumn{3}{|l|}{ Relationship with boy/girlfriends } \\
\hline Good & 152 & 45.6 \\
\hline Bad & 57 & 17.1 \\
\hline Normal & 124 & 37.2 \\
\hline \multicolumn{3}{|l|}{ Current accommodation } \\
\hline Family home & 11 & 2.4 \\
\hline University dormitory & 411 & 89.2 \\
\hline Rented premises & 36 & 7.8 \\
\hline Other & 3 & 0.7 \\
\hline \multicolumn{3}{|l|}{ Grading Point Average (GPA) } \\
\hline Grade A & 49 & 14.3 \\
\hline Grade $\mathrm{B}+$ & 100 & 29.2 \\
\hline Grade B & 125 & 36.5 \\
\hline Grade $\mathrm{C}+$ & 61 & 17.8 \\
\hline Grade C & 7 & 2.0 \\
\hline
\end{tabular}

Regarding the information on student's family, most student's parents were living together (87.4 $\%$ ), often paying attention to their children's study and life $(76.6 \%)$, providing everything needed for their children's learning (89.9\%), and expecting their children's success in the study (92.4\%). During the COVID-19 epidemic, students felt happy to live with their family (98.3 \%), had a good, friendly environment at school $(72.6 \%)$, and tense feelings during the study $(29.3 \%)$. The percentage of satisfaction with the teaching method was $69.1 \%$ (Table 2). 
Table 2 Descriptive statistics for family characteristics and feelings of students during the learning process at school.

\begin{tabular}{|c|c|c|}
\hline Variables & $\begin{array}{l}\text { Frequency } \\
\text { (n) }\end{array}$ & $\begin{array}{c}\text { Percentage } \\
(\%)\end{array}$ \\
\hline \multicolumn{3}{|l|}{ Marital status of parent } \\
\hline Living together & 397 & 87.4 \\
\hline Divorce & 28 & 6.2 \\
\hline Separation & 18 & 4.0 \\
\hline Other & 11 & 2.4 \\
\hline \multicolumn{3}{|l|}{$\begin{array}{l}\text { A family providing everything } \\
\text { needed for learning }\end{array}$} \\
\hline Completely enough & 208 & 45.4 \\
\hline Partially enough & 204 & 44.5 \\
\hline Not enough & 46 & 10.0 \\
\hline \multicolumn{3}{|l|}{$\begin{array}{l}\text { Parent's attention in student's } \\
\text { study and life }\end{array}$} \\
\hline Very often & 202 & 43.6 \\
\hline Often & 153 & 33 \\
\hline Occasionally & 104 & 22.5 \\
\hline No idea & 4 & 0.9 \\
\hline \multicolumn{3}{|l|}{$\begin{array}{l}\text { Parent's expectation about } \\
\text { student's success }\end{array}$} \\
\hline Very expectant & 245 & 52.9 \\
\hline Moderately expectant & 183 & 39.5 \\
\hline Not expectant & 30 & 6.5 \\
\hline No idea & 5 & 1.1 \\
\hline \multicolumn{3}{|l|}{ Feeling happy to live with family } \\
\hline Very happy & 355 & 77.2 \\
\hline Little happy & 97 & 21.1 \\
\hline No idea & 8 & 1.7 \\
\hline \multicolumn{3}{|l|}{$\begin{array}{l}\text { Good and friendly education } \\
\text { environments }\end{array}$} \\
\hline Yes & 336 & 72.6 \\
\hline No & 16 & 3.5 \\
\hline No idea & 111 & 24 \\
\hline \multicolumn{3}{|l|}{ Teachers willing to help students } \\
\hline Yes & 318 & 68.8 \\
\hline No & 25 & 5.4 \\
\hline No idea & 119 & 25.7 \\
\hline \multicolumn{3}{|l|}{ Satisfying teaching methods } \\
\hline Yes & 319 & 69.1 \\
\hline No & 26 & 5.6 \\
\hline No idea & 117 & 25.3 \\
\hline \multicolumn{3}{|l|}{ Feelings about school activities } \\
\hline Comfortable & 286 & 61.7 \\
\hline Uncomfortable & 17 & 3.7 \\
\hline No idea & 160 & 34.6 \\
\hline \multicolumn{3}{|l|}{$\begin{array}{l}\text { Tense feeling during the course } \\
\text { study }\end{array}$} \\
\hline Yes & 135 & 29.3 \\
\hline No & 183 & 39.7 \\
\hline No idea & 143 & 31.0 \\
\hline
\end{tabular}




\section{Worry in COVID-19 context and prevalence of DAS}

In the context of the COVID-19 epidemic, the "very worried" level accounted for $>20 \%$ regarding the worry about beloved one's death (53\%), a tough time for the country's economy (33.2\%), inability to pay bills $(31.2 \%)$, beloved one's unemployment status (29.7\%), overloaded health system $(24.7 \%)$, beloved one's health status (21.6\%), and unable visits to beloved ones (20.7\%) (Table 3). In Table 4, there is a description of depression, anxiety, and stress levels to indicate that $24.8,31$, and $18.8 \%$ of undergraduate public health students experienced depression, anxiety, and stress, respectively. Severe and extremely severe scores for depression, anxiety, and stress were reported in $4.3,6.8$, and $4.6 \%$ of students, respectively.

Table 3 Percentage of worry responses to COVID-19 epidemic.

\begin{tabular}{lccccc}
\hline \multicolumn{1}{c}{$\begin{array}{c}\text { Worry about COVID-19 context } \\
\text { Part D of questionnaire (D1-D14) }\end{array}$} & $\begin{array}{c}\text { At ease } \\
\text { \% }\end{array}$ & $\begin{array}{c}\text { Not } \\
\text { worried } \\
\text { \% }\end{array}$ & $\begin{array}{c}\text { Slightly } \\
\text { nervous } \\
\text { \% }\end{array}$ & $\begin{array}{c}\text { Worried } \\
\text { \% }\end{array}$ & $\begin{array}{c}\text { Very } \\
\text { worried } \\
\text { \% }\end{array}$ \\
\hline D1. Losing someone I love & 18.6 & 7.1 & 8.7 & 12.6 & 53.0 \\
D2. Health system being overloaded & 7.1 & 20.3 & 17.5 & 30.3 & 24.7 \\
D3. My own mental health & 26.0 & 20.1 & 26.8 & 15.4 & 11.7 \\
D4. My own physical health & 21.4 & 24.2 & 24.2 & 18.8 & 11.3 \\
D5. My loved ones'health & 18.6 & 18.6 & 20.1 & 21.0 & 21.6 \\
D6. Restricted liberty of movement & 21.0 & 25.5 & 23.2 & 18.6 & 11.7 \\
D7. Loosing vacation opportunities & 15.4 & 28.4 & 28.1 & 12.6 & 15.6 \\
D8. Small companies running out of business & 14.7 & 23.6 & 29.2 & 19.7 & 12.8 \\
D9. Economic recession in my country & 10.2 & 16.1 & 17.8 & 22.8 & 33.2 \\
D10. Restricted access to food supplies & 12.6 & 22.3 & 23.4 & 25.1 & 16.7 \\
D11. My loved ones becoming unemployed & 11.9 & 15.4 & 18.8 & 24.2 & 29.7 \\
D12. Not being able to pay my bills & 11.3 & 16.2 & 14.9 & 26.4 & 31.2 \\
D13. Not be able to visit beloved one & 16.3 & 17.4 & 26.7 & 18.9 & 20.7 \\
D 14. Decision not to participate in an event which & 22.9 & 22.7 & 22.9 & 18.6 & 12.8 \\
family or friends expect me to attend & & & & & \\
\hline
\end{tabular}

Table 4 Classifications of depression, anxiety, and stress levels among public health students.

\begin{tabular}{lccc}
\hline \multicolumn{1}{c}{ Classification } & $\begin{array}{c}\text { Depression } \\
\mathbf{N}(\boldsymbol{\%})\end{array}$ & $\begin{array}{c}\text { Anxiety } \\
\mathbf{N}(\boldsymbol{\%})\end{array}$ & $\begin{array}{c}\text { Stress } \\
\mathbf{N}(\%)\end{array}$ \\
\hline 1.Normal & $348(75.2)$ & $303(69)$ & $376(81.2)$ \\
2.Mild & $60(13)$ & $38(8.7)$ & $40(8.6)$ \\
3.Moderate & $35(7.6)$ & $68(15.5)$ & $26(5.6)$ \\
4.Severe & $9(1.9)$ & $22(5)$ & $16(3.5)$ \\
5.Extremely severe & $11(2.4)$ & $8(1.8)$ & $5(1.1)$ \\
6.Prevalence (from mild to extremely severe) & $115(24.8)$ & $136(31)$ & $87(18.8)$ \\
Total & $463(100)$ & $439(100)$ & $463(100)$ \\
\hline
\end{tabular}




\section{Factors related to depression, anxiety, and stress}

Depression, anxiety, and stress variables were tested whether to assume the normal distribution by KS test and the null hypothesis was rejected with a $\mathrm{p}$-value $<0.05$. Therefore, the nonparametric tests were used to include Mann Whitney $U$ test and the Kruskal-Wallis test by using the ranks of data. The result in Table 5 revealed the statistically significant differences of depression, anxiety, and stress scores between groups of the year of study, relationships with close friends, and boy/girlfriends, parent's marital status, parent's attention to student's study and life, student's feelings in living with families, and in the educational environment $(\mathrm{p}<0.05)$. The statistically significant differences of both depression and anxiety scores were recognized between groups in terms of tense feelings during learning $(p<0.01)$; differences of both anxiety and stress scores between groups of parent's expectation $(\mathrm{p}<0.05$ and $\mathrm{p}<$ $0.01)$; difference of depression between groups of family's provision $(\mathrm{p}<0.05)$, and difference of stress scores between groups of specialized majors $(\mathrm{p}<0.05)$.

Table 5 The mean rank difference of DAS scores between groups.

\begin{tabular}{|c|c|c|c|c|c|c|c|c|c|}
\hline \multirow{2}{*}{$\begin{array}{c}\text { Characteristics of students } \\
\text { and families }\end{array}$} & \multicolumn{3}{|c|}{ Depression } & \multicolumn{3}{|c|}{ Anxiety } & \multicolumn{3}{|c|}{ Stress } \\
\hline & $\begin{array}{l}\text { Mean } \\
\text { rank }\end{array}$ & $\mathbf{N}$ & $\begin{array}{c}\chi^{2} \\
\text { or } \mathbf{Z}\end{array}$ & $\begin{array}{c}\text { Mean } \\
\text { rank }\end{array}$ & $\mathbf{N}$ & $\begin{array}{c}\chi^{2} \\
\text { or } \mathbf{Z}\end{array}$ & $\begin{array}{c}\text { Mean } \\
\text { rank }\end{array}$ & $\mathbf{N}$ & $\begin{array}{c}\chi^{2} \\
\text { or } \mathbf{Z}\end{array}$ \\
\hline $\begin{array}{r}\text { Male } \\
\text { Female }\end{array}$ & $\begin{array}{l}236.76 \\
230.24\end{array}$ & 461 & $\begin{array}{c}\mathbf{Z} \\
-0.343\end{array}$ & $\begin{array}{l}230.31 \\
231.09\end{array}$ & 461 & $\begin{array}{c}\mathbf{Z} \\
-0.041\end{array}$ & $\begin{array}{l}227.72 \\
231.43\end{array}$ & 461 & $\begin{array}{c}\mathbf{Z} \\
-0.19\end{array}$ \\
\hline $\begin{array}{l}1^{\text {st }} \text {-year } \\
2^{\text {nd }}-\text { year } \\
3^{\text {rd }} \text {-year } \\
4^{\text {th-year }}\end{array}$ & $\begin{array}{l}204.38 \\
256.02 \\
245.78 \\
224.29\end{array}$ & 461 & $\begin{array}{c}\chi^{2} \\
11.82 * *\end{array}$ & $\begin{array}{l}194.27 \\
270.44 \\
243.04 \\
231.83\end{array}$ & 461 & $\begin{array}{c}\chi^{2} \\
21.79 * *\end{array}$ & $\begin{array}{l}187.73 \\
267.95 \\
255.77 \\
223.86\end{array}$ & 461 & $\begin{array}{c}\chi^{2} \\
29.07 * *\end{array}$ \\
\hline $\begin{array}{r}\text { Specialized majors } \\
\text { Community public health } \\
\text { Occupational health } \\
\text { Environmental health }\end{array}$ & $\begin{array}{l}163.48 \\
150.45 \\
159.18\end{array}$ & 318 & $\begin{array}{c}\chi^{2} \\
1.094\end{array}$ & $\begin{array}{l}163.34 \\
152.87 \\
156.69\end{array}$ & 318 & $\begin{array}{c}\chi^{2} \\
0.778\end{array}$ & $\begin{array}{l}168.22 \\
135.11 \\
164.21\end{array}$ & 318 & $\begin{array}{c}\chi^{2} \\
7.18 *\end{array}$ \\
\hline $\begin{array}{l}\text { Relationship with close } \\
\text { friends } \\
\text { Good } \\
\text { Bad } \\
\text { Normal }\end{array}$ & $\begin{array}{l}217.85 \\
111.25 \\
265.79 \\
\end{array}$ & 446 & $\begin{array}{c}\chi^{2} \\
8.669^{*}\end{array}$ & $\begin{array}{c}219.6 \\
82 \\
254.93\end{array}$ & 446 & $\begin{array}{c}\chi^{2} \\
6.275^{*}\end{array}$ & $\begin{array}{c}216.53 \\
151.5 \\
273.34\end{array}$ & 446 & $\begin{array}{c}\chi^{2} \\
10.4^{* *}\end{array}$ \\
\hline $\begin{array}{l}\text { Relationship with } \\
\text { boy/girlfriends }\end{array}$ & $\begin{array}{l}161.09 \\
202.66 \\
157.86\end{array}$ & 333 & $\begin{array}{c}\chi^{2} \\
9.768 * *\end{array}$ & $\begin{array}{l}160.47 \\
205.32 \\
157.39\end{array}$ & 333 & $\begin{array}{c}\chi^{2} \\
11.17^{* *}\end{array}$ & $\begin{array}{l}152.32 \\
198.58 \\
170.48\end{array}$ & 333 & $\begin{array}{c}\chi^{2} \\
9.93 * *\end{array}$ \\
\hline $\begin{array}{r}\text { Current accommodation } \\
\text { Family home } \\
\text { University dormitory } \\
\text { Rented premises } \\
\text { Other }\end{array}$ & $\begin{array}{c}263.45 \\
232.94 \\
199.15 \\
228\end{array}$ & 461 & $\begin{array}{c}\chi^{2} \\
2.88\end{array}$ & $\begin{array}{c}216.5 \\
234.21 \\
191.82 \\
314.67\end{array}$ & 461 & $\begin{array}{c}\chi^{2} \\
4.75\end{array}$ & $\begin{array}{l}179.73 \\
234.93 \\
197.69 \\
279.83\end{array}$ & 461 & $\begin{array}{c}\chi^{2} \\
4.69\end{array}$ \\
\hline $\begin{array}{l}\text { Grade Point Average (GPA) } \\
\text { Last semester } \\
\qquad \text { Grade A } \\
\text { Grade B+ } \\
\text { Grade B } \\
\text { Grade C+ } \\
\text { Grade C }\end{array}$ & $\begin{array}{c}241.2 \\
223.48 \\
230.31 \\
237.7 \\
263.5\end{array}$ & 460 & $\begin{array}{c}\chi^{2} \\
1.297\end{array}$ & $\begin{array}{c}213.11 \\
219.77 \\
241.01 \\
264.04 \\
259\end{array}$ & 460 & $\begin{array}{c}\chi^{2} \\
6.581\end{array}$ & $\begin{array}{c}208.79 \\
229.43 \\
233.71 \\
258.1 \\
313.5\end{array}$ & 460 & $\begin{array}{c}\chi^{2} \\
5.01\end{array}$ \\
\hline
\end{tabular}




\begin{tabular}{|c|c|c|c|c|c|c|c|c|c|}
\hline \multirow{2}{*}{$\begin{array}{c}\text { Characteristics of students } \\
\text { and families }\end{array}$} & \multicolumn{3}{|c|}{ Depression } & \multicolumn{3}{|c|}{ Anxiety } & \multicolumn{3}{|c|}{ Stress } \\
\hline & $\begin{array}{c}\text { Mean } \\
\text { rank }\end{array}$ & $\mathbf{N}$ & $\begin{array}{c}\chi^{2} \\
\text { or } Z\end{array}$ & $\begin{array}{c}\text { Mean } \\
\text { rank }\end{array}$ & $\mathbf{N}$ & $\begin{array}{c}\chi^{2} \\
\text { or } Z\end{array}$ & $\begin{array}{c}\text { Mean } \\
\text { rank }\end{array}$ & $\mathbf{N}$ & $\begin{array}{c}\chi^{2} \\
\text { or } Z\end{array}$ \\
\hline $\begin{array}{r}\text { Marital status of parents } \\
\text { Living together } \\
\text { Divorce } \\
\text { Separation } \\
\text { Other }\end{array}$ & $\begin{array}{l}221.97 \\
296.64 \\
277.19 \\
169.91 \\
\end{array}$ & 454 & $\begin{array}{c}\chi^{2} \\
13.6^{* * *}\end{array}$ & $\begin{array}{c}221.31 \\
303.59 \\
280.5 \\
170.5 \\
\end{array}$ & 454 & $\begin{array}{c}\chi^{2} \\
15.61 * *\end{array}$ & $\begin{array}{l}221.41 \\
323.41 \\
242.33 \\
179.05 \\
\end{array}$ & 454 & $\begin{array}{c}\chi^{2} \\
17.75^{* *}\end{array}$ \\
\hline $\begin{array}{l}\text { Family providing everything } \\
\text { needed for learning } \\
\text { Completely enough } \\
\text { Partially enough } \\
\text { Not enough }\end{array}$ & $\begin{array}{l}227.57 \\
242.29 \\
181.49 \\
\end{array}$ & 458 & $8.25^{*}$ & $\begin{array}{l}221.94 \\
243.69 \\
200.76 \\
\end{array}$ & 458 & 5.29 & $\begin{array}{l}223.76 \\
239.25 \\
212.24 \\
\end{array}$ & 458 & 2.307 \\
\hline $\begin{array}{l}\text { Parent's attention to student's } \\
\text { study and life } \\
\text { Very often } \\
\text { Often } \\
\text { Occasionally } \\
\text { No idea }\end{array}$ & $\begin{array}{c}207.18 \\
227.92 \\
285.09 \\
261 \\
\end{array}$ & 463 & $\begin{array}{c}\chi^{2} \\
24.39 * *\end{array}$ & $\begin{array}{l}208.77 \\
230.08 \\
280.94 \\
206.13\end{array}$ & 463 & $\begin{array}{c}\chi^{2} \\
20.58 * *\end{array}$ & $\begin{array}{c}206.48 \\
240.63 \\
269.66 \\
211.5 \\
\end{array}$ & 463 & $\begin{array}{c}\chi^{2} \\
16.5^{* *}\end{array}$ \\
\hline $\begin{array}{r}\text { Parent's expectation about } \\
\text { student's success } \\
\text { Very expectant } \\
\text { Moderately expectant } \\
\text { Not expectant } \\
\text { No idea }\end{array}$ & $\begin{array}{c}235.73 \\
224.72 \\
232.98 \\
309.8\end{array}$ & 463 & $\begin{array}{l}\chi^{2} \\
2.5\end{array}$ & $\begin{array}{c}248.78 \\
209.98 \\
234.67 \\
200\end{array}$ & 463 & $\begin{array}{c}\chi^{2} \\
9.285^{*}\end{array}$ & $\begin{array}{c}251.48 \\
210.79 \\
194.52 \\
279\end{array}$ & 463 & $\begin{array}{c}\chi^{2} \\
12.9 * *\end{array}$ \\
\hline $\begin{array}{r}\text { Feelings in living with family } \\
\text { Very happy } \\
\text { Little happy } \\
\text { No idea }\end{array}$ & $\begin{array}{l}212.64 \\
289.76 \\
304.44\end{array}$ & 460 & $\begin{array}{c}\chi^{2} \\
29.01 * *\end{array}$ & $\begin{array}{l}216.97 \\
277.96 \\
255.31\end{array}$ & 460 & $\begin{array}{c}\chi^{2} \\
16.63 * *\end{array}$ & $\begin{array}{l}217.17 \\
277.76 \\
248.81\end{array}$ & 460 & $\begin{array}{c}\chi^{2} \\
16.15^{* *}\end{array}$ \\
\hline $\begin{array}{l}\text { Good and friendly education } \\
\text { environments } \\
\text { Yes } \\
\text { No } \\
\text { No idea }\end{array}$ & $\begin{array}{l}214.96 \\
274.84 \\
277.41\end{array}$ & 463 & $\begin{array}{c}\chi^{2} \\
20.5^{* *}\end{array}$ & $\begin{array}{l}213.46 \\
236.09 \\
287.54\end{array}$ & 463 & $\begin{array}{c}\chi^{2} \\
26.09 * *\end{array}$ & $\begin{array}{l}219.17 \\
246.56 \\
268.75\end{array}$ & 463 & $\begin{array}{c}\chi^{2} \\
11.79 * *\end{array}$ \\
\hline $\begin{array}{l}\text { Teachers willing to help } \\
\text { students } \\
\qquad \begin{array}{r}\text { Yes } \\
\text { No } \\
\text { No idea }\end{array} \\
\end{array}$ & $\begin{array}{l}228.23 \\
266.88 \\
232.81 \\
\end{array}$ & 462 & $\begin{array}{c}\chi^{2} \\
2.018\end{array}$ & $\begin{array}{l}226.79 \\
242.92 \\
241.68 \\
\end{array}$ & 462 & $\begin{array}{c}\chi^{2} \\
1.295\end{array}$ & $\begin{array}{l}232.68 \\
248.54 \\
224.76 \\
\end{array}$ & 462 & $\begin{array}{c}\chi^{2} \\
0.744\end{array}$ \\
\hline $\begin{array}{r}\text { Satisfying teaching methods } \\
\text { Yes } \\
\text { No } \\
\text { No idea }\end{array}$ & $\begin{array}{l}226.6 \\
259.8 \\
238.5\end{array}$ & 462 & 1.98 & $\begin{array}{l}228.39 \\
222.98 \\
241.86\end{array}$ & 462 & 1.0 & $\begin{array}{l}228.28 \\
257.85 \\
234.43\end{array}$ & 462 & $\begin{array}{c}\chi^{2} \\
1.26\end{array}$ \\
\hline $\begin{array}{l}\text { Feelings about school } \\
\text { activities } \\
\text { Comfortable } \\
\text { Uncomfortable } \\
\text { No idea }\end{array}$ & $\begin{array}{l}233.03 \\
273.47 \\
225.75\end{array}$ & 463 & $\begin{array}{c}\chi^{2} \\
2.06\end{array}$ & $\begin{array}{l}231.3 \\
262.4 \\
230\end{array}$ & 463 & $\begin{array}{c}\chi^{2} \\
0.941\end{array}$ & $\begin{array}{l}231.06 \\
246.12 \\
232.18\end{array}$ & 463 & $\begin{array}{c}\chi^{2} \\
0.206\end{array}$ \\
\hline $\begin{array}{l}\text { Tense feeling during the } \\
\text { course study } \\
\qquad \text { Yes } \\
\text { No } \\
\text { No idea }\end{array}$ & $\begin{array}{c}267.73 \\
199.21 \\
237\end{array}$ & 461 & $\begin{array}{c}\chi^{2} \\
21.63 * *\end{array}$ & $\begin{array}{c}245.69 \\
203.83 \\
251.9\end{array}$ & 461 & $\begin{array}{c}\chi^{2} \\
13.02 * *\end{array}$ & $\begin{array}{l}234.41 \\
217.96 \\
244.47\end{array}$ & 461 & $\begin{array}{c}\chi^{2} \\
3.34\end{array}$ \\
\hline
\end{tabular}

Note: $* \mathrm{p}<0.05 ; * * \mathrm{p}<0.01$. 


\section{Predictors of depression, anxiety, and stress in public health students}

In the bivariate analysis, the non-parametric tests were used to determine the associations between variables and DAS. The variables that had statistically significant associations with DAS at the 0.05 level in the bivariate analysis were further tested using ordinal logistic regression analysis to identify predictors of DAS in Table 6.

Table 6 Ordinal logistic regression model for predicting depression, anxiety, and stress in public health students.

\begin{tabular}{|c|c|c|c|c|c|c|}
\hline \multirow[b]{2}{*}{ Predictors } & \multicolumn{2}{|c|}{ Depression } & \multicolumn{2}{|c|}{ Anxiety } & \multicolumn{2}{|c|}{ Stress } \\
\hline & $\begin{array}{c}\text { Wald } \\
\text { (df) }\end{array}$ & $\begin{array}{c}\text { OR } \mathbf{R}_{\text {adj }} \\
(95 \% \text { CI })\end{array}$ & $\begin{array}{l}\text { Wald } \\
\text { (df) }\end{array}$ & $\begin{array}{c}\text { ORadj } \\
(95 \% \text { CI })\end{array}$ & $\begin{array}{c}\text { Wald } \\
\text { (df) }\end{array}$ & $\begin{array}{c}\text { OR }_{\text {adj }} \\
(95 \% \text { CI })\end{array}$ \\
\hline $\begin{array}{l}1^{\text {st }} \text {-year } \\
2^{\text {nd }} \text {-year } \\
3^{\text {rd }} \text {-year } \\
4^{\text {th }} \text {-year }\end{array}$ & $\begin{array}{l}2.09(1) \\
0.06(1) \\
0.01(1)\end{array}$ & $\begin{array}{c}0.5(0.2-1.2) \\
1.1(0.5-2.3) \\
1.04(0.5-2.1) \\
1\end{array}$ & $\begin{array}{c}1.62(1) \\
2.45(1) \\
0.006 \\
(1)\end{array}$ & $\begin{array}{c}0.57(0.2-1.3) \\
1.82(0.8-3.8) \\
1.02(0.5-2.1) \\
1\end{array}$ & $\begin{array}{l}0.1(1) \\
6.7(1) \\
1.5(1)\end{array}$ & $\begin{array}{c}1.3(0.2-6.5) \\
2.7(1.2-6) \\
1.6(0.7-3.4) \\
1\end{array}$ \\
\hline $\begin{array}{l}\text { Specialized majors } \\
\text { Community public health } \\
\text { Occupational health } \\
\text { Environmental health }\end{array}$ & & & & & $\begin{array}{l}0.06(1) \\
7.2 *(1)\end{array}$ & $\begin{array}{c}0.9(0.4-1.9) \\
0.2(0.1-0.7) \\
1\end{array}$ \\
\hline $\begin{array}{l}\text { Relationship with close } \\
\text { friends } \\
\text { Good } \\
\text { Bad } \\
\text { Normal }\end{array}$ & $\begin{array}{l}0.02(1) \\
0.59(1)\end{array}$ & $\begin{array}{c}0.9(0.4-1.8) \\
0.2(0.01-6.4) \\
1\end{array}$ & $\begin{array}{l}0.01(1) \\
1.83(1)\end{array}$ & $\begin{array}{c}0.95(0.5-1.8) \\
0.1(0.005-2.5) \\
1\end{array}$ & $\begin{array}{c}1.7(1) \\
3.74(1)\end{array}$ & $\begin{array}{c}0.6(0.2-1.2) \\
0.02(0-1) \\
1\end{array}$ \\
\hline $\begin{array}{l}\text { Relationship with } \\
\text { boyfriends/girlfriends } \\
\text { Good } \\
\text { Bad } \\
\text { Normal }\end{array}$ & $\begin{array}{c}0.28(1) \\
7.19 *(1)\end{array}$ & $\begin{array}{l}0.8(0.5-1.4) \\
2.5(1.2-4.9) \\
1\end{array}$ & $\begin{array}{l}0.73(1) \\
2.02(1)\end{array}$ & $\begin{array}{l}0.8(0.5-1.3) \\
1.6(0.8-3.1) \\
1\end{array}$ & $\begin{array}{l}4.9 *(1) \\
0.01(1)\end{array}$ & $\begin{array}{c}0.5(0.2-0.9) \\
0.9(0.4-2.0) \\
1\end{array}$ \\
\hline $\begin{array}{r}\text { Marital status of parents } \\
\text { Living together } \\
\text { Divorce } \\
\text { Separation } \\
\text { Other (Widow) }\end{array}$ & $\begin{array}{c}0.60(1) \\
4.55^{*}(1) \\
0.08(1)\end{array}$ & $\begin{aligned} & 2.5(0.2-27.1) \\
& 15.5(1.2-192.3) \\
& 1.4(0.1-19.3) \\
& 1\end{aligned}$ & $\begin{array}{l}0.90(1) \\
1.96(1) \\
0.29(1)\end{array}$ & $\begin{array}{c}2.7(0.3-22.4) \\
5.0(0.5-47.6) \\
1.9(0.1-19.6) \\
1\end{array}$ & $\begin{array}{l}1.2(1) \\
3.45(1) \\
0.13(1)\end{array}$ & $\begin{array}{c}4.2(0.3-57) \\
13(0.8-218) \\
0.5(0.03-10) \\
1\end{array}$ \\
\hline
\end{tabular}

\begin{tabular}{|c|c|c|c|c|c|c|}
\hline $\begin{array}{l}\text { Family providing } \\
\text { everything needed for } \\
\text { learning } \\
\text { Completely enough } \\
\text { Partially enough } \\
\text { Not enough }\end{array}$ & $\begin{array}{l}6.47 *(1) \\
4.95 *(1)\end{array}$ & $\begin{array}{l}3.1(1.3-7.6) \\
2.6(1.1-6.2) \\
1\end{array}$ & & & & \\
\hline $\begin{array}{l}\text { Parent's expectation about } \\
\text { student's success } \\
\text { Very expectant } \\
\text { Moderately expectant } \\
\text { Not expectant } \\
\text { No idea }\end{array}$ & & & $\begin{array}{l}1.12(1) \\
0.08(1) \\
0.17(1)\end{array}$ & $\begin{array}{c}3.3(0.3-32.3) \\
1.3(0.1-12.9) \\
1.6(0.1-16.7) \\
1\end{array}$ & $\begin{array}{l}0.1(1) \\
0.03(1) \\
0.06(1)\end{array}$ & $\begin{array}{c}1.8(0.04-155) \\
0.6(0.01-57) \\
1.8(0.02-155) \\
1\end{array}$ \\
\hline $\begin{array}{l}\text { Parent's attention to } \\
\text { student's study and life } \\
\text { Very often }\end{array}$ & $0.15(1)$ & $1.6(0.1-22.3)$ & $0.54(1)$ & $2.6(0.1-37)$ & $2.45(1)$ & $0.52(0.23-1.2)$ \\
\hline
\end{tabular}




\begin{tabular}{|c|c|c|c|c|c|c|}
\hline \multirow[b]{2}{*}{ Predictors } & \multicolumn{2}{|c|}{ Depression } & \multicolumn{2}{|c|}{ Anxiety } & \multicolumn{2}{|c|}{ Stress } \\
\hline & $\begin{array}{c}\text { Wald } \\
\text { (df) }\end{array}$ & $\begin{array}{c}\text { ORadj } \\
(95 \% \mathrm{CI}) \\
\end{array}$ & $\begin{array}{l}\text { Wald } \\
\text { (df) }\end{array}$ & $\begin{array}{c}\text { ORadj } \\
(95 \% \mathrm{CI}) \\
\end{array}$ & $\begin{array}{c}\text { Wald } \\
\text { (df) }\end{array}$ & $\begin{array}{c}\text { ORadj } \\
(95 \% \mathrm{CI}) \\
\end{array}$ \\
\hline Often & $0.39(1)$ & $2.3(0.1-31.6)$ & $1.19(1)$ & $4.4(0.3-63)$ & $2.05(1)$ & $0.57(0.26-1.1)$ \\
\hline Occasionally & $0.94(1)$ & $3.7(0.2-52.9)$ & $1.10(1)$ & $4.2(0.2-62)$ & & 1 \\
\hline No idea & & 1 & & 1 & & \\
\hline \multicolumn{7}{|l|}{$\begin{array}{l}\text { Feelings of living with } \\
\text { family }\end{array}$} \\
\hline Very happy & $1.19(1)$ & $0.3(0.07-2.1)$ & $1.70(1)$ & $0.32(0.06-1.7)$ & $0.312(1)$ & $0.45(0.02-7.1)$ \\
\hline Happy & $0.26(1)$ & $0.6(0.1-3.5)$ & $0.99(1)$ & $0.42(0.07-2.3)$ & $0.109(1)$ & $0.63(0.04-9.4)$ \\
\hline No idea & & 1 & & 1 & & 1 \\
\hline \multicolumn{7}{|l|}{$\begin{array}{l}\text { Good and friendly school } \\
\text { environments }\end{array}$} \\
\hline Yes & $10.1 *(1)$ & $0.35(0.1-0.6)$ & $8.7 *(1)$ & $0.3(0.2-0.7)$ & $2.69(1)$ & $0.54(0.2-1)$ \\
\hline No & $0.003(1)$ & $1.03(0.3-3.5)$ & $1.1(1)$ & $0.5(0.1-1.7)$ & $0.60(1)$ & $2(0.3-11)$ \\
\hline No idea & & 1. & & 1 & & 1 \\
\hline \multicolumn{7}{|l|}{$\begin{array}{l}\text { Tense feeling during the } \\
\text { course study }\end{array}$} \\
\hline Yes & $16.3 *(1)$ & $3.57(1.9-6.6)$ & $2.86(1)$ & $1.6(0.9-3.0)$ & & \\
\hline No & $0.02(1)$ & $1.04(0.5-1.8)$ & $5.51 *(1)$ & $0.5(0.2-0.8)$ & & \\
\hline No idea & & 1 & & 1 & & \\
\hline $\begin{array}{l}\text { D2. Health system being } \\
\text { overloaded }\end{array}$ & & & $\begin{array}{c}4.61 * \\
(1)\end{array}$ & $1.3(1.02-1.8)$ & & \\
\hline $\begin{array}{l}\text { D4. My own physical } \\
\text { health }\end{array}$ & $4.3 *(1)$ & $1.36(1.01-1.84)$ & $\begin{array}{c}6.46^{*} \\
(1)\end{array}$ & $1.5(1.1-2.1)$ & & \\
\hline $\begin{array}{l}\text { D6. Restricted liberty of } \\
\text { movement }\end{array}$ & & & & & $10.8 *(1)$ & $1.7(1.2-2.4)$ \\
\hline
\end{tabular}

$* \mathrm{p}<0.05$.

\section{Depression}

The predictors of depression identified in the ordinal logistic regression could comprise relationship with boy/girlfriends, marital status of parents, family provision for learning, feelings about educational environments, tense feeling during learning, and "Worry about my physical health during COVID-19 epidemic". For the relationship with boy/girlfriends, the odds ratio of students having a bad relationship to experience depression was 2.5 times that of students having a normal relationship $(\mathrm{OR}=2.5 ; 95 \%$ CI:1.2- 4.9; $\mathrm{p}<0.05)$. Compared with students whose father/mother was a widow, the odds ratio of depression was 15.5 (95\% CI:1.2 -192.3; p < 0.05) for those whose parents were divorced. Compared with students who did not have enough family's provision for learning, the odds ratio of depression was $3.1(95 \%$ CI:1.3 -7.6; p < 0.05), and 2.6 (95\%CI:1.1 -6.2; p < 0.05) for those who had completely enough and partially enough family's provision, respectively.Compared with students who had no idea for an education environment, and for feelings during learning, the odds ratio of depression was 0.35 (95\% CI:0.1 - 0.6; p < 0.05), and $3.57(95 \% \mathrm{CI}: 1.9-6.6 ; \mathrm{p}<0.05)$ for those who agreed on the good and friendly education environment and had a tense feeling during learning, respectively. During the COVID19 epidemic, an increase in worry level about "my physical health" from the $1^{\text {st }}$ level "At ease"to the $5^{\text {th }}$ level "Very worried"was associated with an increase in the odds of depression, with an odds ratio of 1.36 (95\% CI:1.01 - 1.84; $\mathrm{p}<0.05)$.

\section{Anxiety}

The statistically significant predictors of anxiety included feelings aboutthe education environment, tense feeling during learningand "Worry about health system being overloaded and my physical health during COVID-19 epidemic". Compared with students who had no idea for the educational environment, 
and for feelings during learning, the odds ratio of anxiety was 0.3 (95\% CI: $0.2-0.7 ; \mathrm{p}<0.05$ ), and 0.5 (95\% CI:0.2-0.8; $<$ < 0.05) for those who agreed on the good and friendly education environment and had no tense feeling during learning, respectively.Then, an increase in worry level about "health system being overloaded" and "my physical health" during COVID-19 was associated with an increase in the odds of anxiety, with an odds ratio of $1.3(95 \% \mathrm{CI}: 1.02-1.8$; $\mathrm{p}<0.05)$, and 1.5 (95\% CI: $1.1-2.1 ; \mathrm{p}<0.05)$, respectively.

\section{Stress}

The statistically significant predictors of stress consisted of specialized majors of public health, and relationship with boyfriend/girlfriends. Occupational health students were 0.2 times less likely to experience stress than environmental health students $(\mathrm{OR}=0.2 ; 95 \% \mathrm{CI}: 0.1-0.7 ; \mathrm{p}<0.05)$. Students in a good relationship with a boyfriend/girlfriend were 0.5 times less likely to experience stress than students in a casual relationship

\section{Discussion}

This $1^{\text {st }}$ study evaluated the psychological impacts of the COVID-19 pandemic on undergraduate public health students who have positions both as students and future public health staff on the frontline to fight COVID-19 in Thailand. Therefore, in the scope of discussion, DAS levels of public health students were compared with those of health profession's students and college or university students in general. In the current study, the prevalence of depression, anxiety, and stress were $24.8 \%$ (mainly mild symptom), $31 \%$ (mainly moderate symptom), and $18.8 \%$ (mainly mild symptom), respectively. Another study in southern China during the early outbreak peak of COVID-19 (upper week of February 2020) revealed that the prevalence of depression and anxiety of college students were 12.2 and $7.7 \%$, respectively [33], lower than the prevalence in our study. The gap in the prevalence of depression and anxiety might be due to the differences in survey time. According to the authors' explanation, the present study was conducted during Chinese New Year when college students turned back home to enjoy their pleasure of vacation. Thus, the anxiety and depression symptoms during this period could be significantly lower than those during other periods [33]. In another study on depression and anxiety of medical students in Beijing and Wuhan - the epicenter of COVID-19 in China, the prevalence of depression was $25.3 \%$ and anxiety was $17.1 \%$ in mid-February 2020 [27]. The distribution rate of depression among Thai public health students and Chinese medical students is nearly the same. However, the prevalence of anxiety in Thai students was almost twice that of Chinese students. The anxiety rate among public health students might be greater due to the survey conducted at the end of a long period of social distancing of nearly 6 months. Moreover, the highest prevalence for anxiety (31\%) compared to depression $(24.8 \%)$ and stress $(18.8 \%)$ in the present study is consistent with the conclusion of Center for Collegiate Mental Health. Anxiety has become the most common mental health problem among college students and showed a consistent increase and consistent with the most common presenting concerns in counseling centers [34]. In gender, DASS-21 scores were not significantly different between male and female public health students, consistent with the results of the study on university students in Bangladesh [35].

For student's depression, the factor of family provision for student's learning was identified as the predictor. Students with full or partial family provision for their studies were 6.47 or 4.95 times more likely to experience depression than students who did not receive sufficient family support. This could explain that during the COVID-19 epidemic, students who did not receive adequate family support probably learned how to manage their living expenses and adapted to difficult circumstances of economic downturns. Therefore, their mental health status is firmer than that of students who have not experienced difficult and complicated situations. With aspects of academic factors such as academic grades and the year of study, both academic factors were not identified as predictors of DAS. The results are consistent with research on DAS among dental students in Europe, Japan and Australia [36-39]. High levels of depression and other mental disorders may not result from academic performance. Our findings indicate that all students experience tense feelings in learning leading to depression and anxiety. Next, the educational environment is a predictor of depression and anxiety in the present study. Students who felt a good and friendly education environment may experience less depression and less anxiety $(\mathrm{OR}=0.35$ and $\mathrm{OR}=0.3$, respectively) compared to others. A friendly education environment implies student's satisfaction with their faculty and peer relationships that were also the most significant and strongest predictors of DAS in dental students [36]. Next, the group of factors related to the personal relationship was considered statistically significant predictors of DAS. Students who had a bad or good relationship with a boy/girlfriend might have more depression or less stress, respectively. Then, the family relationship including the marital status of parents might influence the close connection and emotional 
bonds between parents and their children (students). This study revealed parent's divorce to be a strong predictor of depression of students compared to another marital status. Hence, factors associated with human relationships seem to have more impacts on psychological health than other academic factors. Finally, worries in the COVID-19 context considered as predictors of DAS consisted of 3 worries. An increase in worries about "My physical health" was associated with increases in the odds of depression and anxiety. Worries about "Health system being overloaded" and "Restricted liberty of movement" were associated with anxiety and stress, respectively. Firstly, worry about the "health system overloaded" during the COVID-19 outbreak can be seen as a typical feature of public health students. They are always concerned about the health system's capacity to deal with the COVID-19 pandemic. This special worry is not recognized in medical and dental students. Secondly, worry about "Restricted liberty of movement", which is considered a predictor of stress, is also consistent with social isolation associated with mental health disorders among university students in France [40]. The review by Brooks et al also pointed out that concern about social isolation and reduced social contact was popular among students confined during the COVID-19 epidemic [24].

As for the limitation of the study, the cross-sectional study design limited the temporal relationship between exposure and outcome, so a causal relationship could not be established.

\section{Conclusions}

After a long period of school closure in Thailand due to COVID-19 outbreaks, the prevalence of depression, anxiety, and stress among undergraduate public health students were 24.8, 31, and $18.8 \%$ recorded from August to October 2020. Protecting the mental health of health profession's students is becoming even more important in the context of a pandemic because they are the future workforce on the frontline to fight COVID-19. The results of the study have identified significant predictors of mental disorders in Thai public health students. Firstly, a good and friendly education environment, which embraces student's satisfaction with their faculty and peer relationships, is a useful predictor to prevent depression and anxiety. Next, the student's tense feelings in learning, which are predictors of depression and anxiety, need to be addressed with the support of peers and faculty. Finally, the university should pay attention to students' issues that affect their emotions considered as predictors, such as parents' divorce, disappointment in love, etc. During the COVID-19 outbreaks, noticeable worries about "My physical health", "Health systems being overloaded", and "Restricted liberty of movement" are also considered predictors of depression, anxiety, and stress. The findings of this study are needed to develop and implement strategies for the prevention and management of depression, anxiety, and stress in public health students. The establishment of psychological support, reduction of growing academic burden with changes of learning needs, and improvement of educational environment should be done.

\section{Acknowledgements}

The authors would like to thank all the public health students at Walailak University who volunteered to participate in this study and would like to express sincere thanks to the Research Institute for Health Science, Walailak University, Thailand, for sponsoring this study (Project number: WU-ECAH-2-239-63).

\section{References}

[1] World Health Organization.Depression and other common mental disorders: Global health estimates, Available at:https://apps.who.int/iris/bitstream/handle/10665/254610/WHO-MSD-MER2017.2-eng.pdf, accessed February 2021.

[2] World Health Organization. Depression, Available at: https://www.who.int/healthtopics/depression\#tab=tab_1, accessed February 2021.

[3] R Bassi, S Sharma and M Kaur. A study of correlation of anxiety levels with body mass index in new MBBS students. Natl. J. Physiol. Pharm. Pharmacol. 2014; 4,208-12.

[4] Medline Plus. Stress and your health, Available at: https://medlineplus.gov/ency/article/003211 .htm\#: :text=Stress\%20is\%20a\%20feeling\%20of,danger\%20or\%20meet\%20a\%20deadline,accesse d February 2021.

[5] N Bayram and N Bilgel. The prevalence and socio-demographic correlations of depression, anxiety and stress among a group of university students.Soc. Psychiatry Psychiatr. Epidemiol. 2008; 43, 667-72. 
[6] LN Dyrbye, MR Thomas and TD Shanafelt. Systematic review of depression, anxiety, and other indicators of psychological distress among U.S. and Canadian medical students. Acad. Med. 2006; 81, 354-73.

[7] A Ali, HM Rao, S Ali, T Ahmed, M Safi, A Malik and B Husan. Prevalence of anxiety and depression and their associated risk factors among engineering students in Karachi, Pakistan. Int. J. Emerg. Tech. Adv. Eng. 2014; 4, 52-5.

[8] AR Abdallah and HM Gabr. Depression, anxiety and stress among first year medical students in an Egyptian public university. Int. Res. J. Med. Med. Sci. 2014; 2, 11-9.

[9] HM Stallman. Prevalence of psychological distress in university students--implications for service delivery. Aust. Fam. Phys. 2008; 37, 673-7.

[10] DP Turner, ME Thompson, LR Huber and AA Arif. Depressive symptoms and academic performance of North Carolina college students. N. C. Med. J. 2012; 73, 169-75.

[11] M Marcus, T Yasamy, MV Ommeren, D Chisholm and S Saxena. Depression: A Global Public Health Concern, Available at: http://www.who.int/mental_health/management/depression/who_paper_depression_wfmh_2012.pdf , accessed February 2021.

[12] AK Ibrahim, SJ Kelly, CE Adams and C Glazebrook. A systematic review of studies of depression prevalence in university students. J. Psychiatr. Res. 2013; 47, 391-400.

[13] BE Wallace, J Masiak and MR Pabis. Depression in medical students: Reviewing its prevalence, risk factors, consequences, and management in order to provide student treatment recommendations for the Polish medical education system. Pol. J. Public Health 2013;123, 259-64.

[14] KO Asante and J Andoh-Arthur. Prevalence and determinants of depressive symptoms among university students in Ghana. J. Affect. Disord. 2015; 171, 161-6.

[15] K Hunt and KN Gable. Prevalence of depressive symptoms and obsessive-compulsive personality traits among pharmacy students. Curr. Pharm. Teach. Learn. 2013; 5, 541-5.

[16] R Puthran, MWB Zhang, WW Tam and RC Ho. Prevalence of depression amongst medical students: A meta-analysis. Med. Educ. 2016; 50, 456-68.

[17] HK Silver and AD Glicken. Medical student abuse: Incidence, severity, and significance. J. Am. Med. Assoc. 1990; 263, 527-32.

[18] PM Niemi and PT Vainiomaki. Medical students' academic distress, coping, and achievement strategies during the preclinical years. Teach. Learn. Med. 1999; 11, 125-34.

[19] A Syed, SS Ali and M Khan. Frequency of depression, anxiety and stress among the undergraduate physiotherapy students.Pak. J. Med. Sci. 2018; 34, 468-71.

[20] Y Han and H Yang. The transmission and diagnosis of 2019 novel coronavirus infection disease (COVID-19): A Chinese perspective. J. Med. Virol. 2020; 92, 639-44.

[21] ML Holshue, CD Bolt, S Lindquist, KH Lofy, J Wiesman, H Bruce, C Spitters, K Ericson, S Wilkerson, A Tural, G Diaz, A Cohn, L Fox, A Patel, SI Gerber, L Kim, S Tong, X Lu, S Lindstrom, MA Pallansch, WC Weldon, HM Biggs, TM Uyeki and SK Pillai. First case of 2019 novel coronavirus in the United States. N. Engl. J. Med. 2020; 382, 929-36.

[22] World Health Organization. Thailand: How a strong health system fights a pandemic, Available at:https://www.who.int/publications/m/item/thailand-how-a-strong-health-system-fights-apandemic, accessed February 2021.

[23] GJ Rubin and S Wessely. The psychological effects of quarantining a city. Br. Med. J. 2020; 368, $\mathrm{m} 313$.

[24] SK Brooks, RK Webster, LE Smith, L Woodland, S Wessely, N Greenberg and GJ Rubin. The psychological impact of quarantine and how to reduce it: Rapid review of the evidence. Lancet 2020; 395, 912-20.

[25] C Ravesloot, B Ward, T Hargrove, J Wong, N Livingston, L Torma and C Ipsen. Why stay home? Temporal association of pain, fatigue and depression with being at home. Disabil. Health J. 2016; 9 , 218-25.

[26] Z Hakami, SB Khanagar, S Vishwanathaiah, A Hakami, AM Bokhari, AH Jabali, D Alasmari and AM Aldrees. Psychological impact of the coronavirus disease 2019 (COVID-19) pandemic on dental students: A nationwide study. J. Dent. Educ. 2020; 85, 494-503.

[27] H Xiao, W Shu, M Li, Z Li, F Tao, X Wu, Y Yu, H Meng, SH Vermund and Y Hu. Social distancing among medical students during the2019 coronavirus disease pandemic in china: Disease awareness, anxiety disorder, depression, and behavioral activities. Int. J. Environ. Res. Public Health 2020; 17, 5047. 
[28] L Sullivan. Power and sample size determination, Available at: https://sphweb.bumc.bu.edu/otlt/ mph-modules/bs/bs704_power/bs704_power_print.html, accessed July 2021.

[29] World Health Organization. Survey tools and guidance: Rapid, simple, flexible behavioural insights on COVID-19, Available at: https://apps.who.int/iris/handle/10665/333549, accessed February 2021.

[30] SH Lovibond and PH Lovibond. Manual of the depression, anxiety, and stress scales. $2^{\text {nd }}$ eds. Psychology Foundation of Australia, Sydney, Australia, 1995.

[31] Psychology Foundation of Australia. Depression, Anxiety, and Stress Scales (DAS), Available at: http://www2.psy.unsw.edu.au/DASS/Thai/DASS21Thai\%20NCHECR.pdf, accessed February 2021.

[32] T Barrientos-Gutierrez, S Martinez-Alcantara and I Mendez. Construct validity, reliability, and cutoff point of the Subjective Symptoms of Fatigue Test in Mexican workers. Salud Publica Mex. 2004; 46, 516-23.

[33] ZH Wang, HL Yang, YQ Yang, D Liu, ZH Li, XR Zhang, YJ Zhang, D Shen, PL Chen, WQ Song, $\mathrm{XM}$ Wang, XB Wu, XF Yang and C Mao. Prevalence of anxiety and depression symptom, and the demands for psychological knowledge and interventions in college students during COVID-19 epidemic: A large cross-sectional study. J. Affect. Disord. 2020; 275, 188-93.

[34] Center for Collegiate Mental Health. Annual Report: Publication, 2015. No. STA 15-30, Available at:https://eric.ed.gov/?id=ED572760, accessed February 2021.

[35] S Islam, R Akter, T Sikder and MD Griffiths. Prevalence and factors associated with depression and anxiety among first-year university students in Bangladesh: A cross-sectional study. Int. J. Ment. Health Addict. 2020. DOI: https://doi.org/10.1007/s11469-020-00242-y.

[36] S Basudan, N Binanzan and A Alhassan. Depression, anxiety and stress in dental students. Int. J. Med. Educ. 2017; 8, 179-86.

[37] R Gorter, R Freeman, S Hammen, H Murtomaa, A Blinkhorn and G Humphris. Psychological stress and health in undergraduate dental students: Fifth year outcomes compared with first year baseline results from five European dental schools. Eur. J. Dent. Educ. 2008; 12, 61-8.

[38] G Sugiura, K Shinada andY Kawaguchi. Psychological well-being and perceptions of stress amongst Japanese dental students. Eur. J. Dent. Educ. 2005; 9, 17-25.

[39] AE Sanders and K Lushington. Effect of perceived stress on student performance in dental school. J. Dent. Educ. 2002; 66, 75-81.

[40] M Wathelet, S Duhem, G Vaiva, T Baubet, E Habran, E Veerapa, C Debien, S Molenda, M Horn, P Grandgenevre, CE Notredame and F D'Hondt. Factors associated with mental health disorders among university students in France confined during the COVID-19 pandemic. JAMA Netw. Open 2020; 3, e2025591. 